\title{
Plantas que acumulan metales, su importancia. Leucocroton havanensis Borhidi hiperacumuladora de níquel
}

\author{
Metal accumulating plants, their importance. Nickel \\ hyperaccumulator Leucocroton havanensis Borhidi
}

\author{
(iD) Dubiel Alfonso González ${ }^{\unrhd}$, (iD) Rolando Reyes Fernández¹, (iDDaymara Rodriguez Alonso \\ (iD) Eduardo Menéndez Álvarez² \\ ${ }^{1}$ Universidad Agraria de La Habana. La Habana, Cuba \\ Universidad Le Cordon Bleu. Lima, Perú
}

\begin{tabular}{llll}
\hline Recibido: $22 / 11 / 2019$ & Revisado: $11 / 01 / 2020$ & Aceptado: 21/02/2020 & Publicado: 18/06/2020 \\
\hline
\end{tabular}

\section{RESUMEN}

Más de 700 especies vegetales en el mundo son capaces de acumular en sus tejidos altas cantidades de elementos metálicos ( $\mathrm{Ni}, \mathrm{Cu}, \mathrm{Co}, \mathrm{Cr}, \mathrm{Mn}$ y $\mathrm{Zn}$, entre otros) por lo que se denominan hiperacumuladoras de metales. Este proceso fisiológico se ha interpretado como una adaptación de las plantas a los suelos metalíferos donde habitan. Leucocroton havanensis Borhidi es una especie hiperacumuladora de Ni endémica del matorral xeromorfo espinoso sobre "suelo serpentina" en Cuba. La naturaleza química de estos suelos metalíferos, debido a las altas concentraciones de $\mathrm{Ni}, \mathrm{Cr}, \mathrm{Co}, \mathrm{Mn}, \mathrm{Mg}$ y Fe, unido a las bajas concentraciones de $\mathrm{Ca}$ y macronutrientes como N, P y K, hacen que las áreas alberguen una alta representación de plantas endémicas con características fisiológicas distintivas. La especie L. havanensis está clasificada como amenazada en la Lista Roja de la Flora de Cuba, debido al reducido número de poblaciones existentes como consecuencia de la actividad económica del hombre. En el contexto de un desarrollo sostenible se han implementado en varios países la fitorremediación y la agrominería. Estas nuevas tecnologías utilizan plantas hiperacumuladoras como vía alternativa para la extracción de metales del suelo. De esta forma se evita el daño al medio ambiente y se conservan los recursos fitogenéticos de las zonas mineras. La función ecológica de la hiperacumulación de metales pudiera estar relacionada con la defensa de estas especies ante patógenos y herbívoros. El Ni hiperacumulado en las hojas de Streptanthus polygaloides protege la planta del ataque de bacterias y hongos y de la babosa Ambigolimax valentianus.

Palabras clave: Hiperacumuladoras, metales, rehabilitación, suelos, plantas.

\begin{abstract}
Over 700 of vegetable species in the world are capable of accumulating in their tissues large amounts of metal elements ( $\mathrm{Ni}, \mathrm{Cu}, \mathrm{Co}, \mathrm{Cr}, \mathrm{Mn}$ and $\mathrm{Zn}$, among others); that is why they are called metal hyperaccumulators. This physiological process has been interpreted as an adaptation of plants to the metal polluted soils where they live. Leucocroton havanensis Borhidi is an endemic Ni hyperaccumulator species of xeromorphic thorn bushes on "serpentine soils" in Cuba. Due to the high concentrations of $\mathrm{Ni}, \mathrm{Cr}, \mathrm{Co}, \mathrm{Mn}, \mathrm{Mg}$ and $\mathrm{Fe}$, together with the low concentrations of $\mathrm{Ca}$
\end{abstract}


and macronutrients such as $\mathrm{N}, \mathrm{P}$, and $\mathrm{K}$, these metal polluted soils host a high representation of endemic plants with distinct physiological characteristics. The $L$. havanensis species is classified as endangered in the Red List of Cuban Flora due to the reduced amount of existing population because of human economic activity. Phytoremediation and agro-mining have been implemented in several countries within the context of sustainable development. These new technologies use hyperaccumulating plants as an alternative way to extract metals from the soil. Damage to the environment is thus avoided and the phytogenetic resources of the mining areas are preserved. The ecological function of metal hyperaccumulation may be related to the defense of these species against pathogens and herbivores. The Hyperaccumulated $\mathrm{Ni}$ in the leaves of Streptanthus polygaloides protect the plant from the attack of bacteria and fungi and from the Ambigolimax valentianus slugs.

Keywords: Hyperaccumulators, metals, rehabilitation, soils, plants.

\section{INTRODUCCIÓN \\ La hiperacumulación de metales pesa- dos por las plantas}

Las plantas que crecen en suelos metalíferos han desarrollado durante la evolución mecanismos fisiológicos para evitar la toxicidad por los metales.Algunas controlan la entrada del metal en la raíz y su transporte a la parte aérea, y otras han desarrollado mecanismos para evitar el efecto nocivo de los iones en las células de las hojas. De acuerdo con estas características, las plantas se clasifican en exclusoras, acumuladoras e indicadoras. Las exclusoras restringen la translocación del metal desde la raíz a la parte aérea, de forma que la concentración encontrada en las hojas es baja. Por el contrario, las acumuladoras son capaces de concentrar el metal en su parte aérea, sin la presencia de síntomas o limitación alguna para su crecimiento, desarrollo y reproducción. En las indicadoras existe una relación proporcional entre la concentración del metal en el suelo, la absorción y la acumulación del metal en la planta, de forma que la concentración en las hojas refleja la del suelo; en muchos casos aparecen síntomas de toxicidad visibles en la planta (Baker, 1981)

El término "hiperacumulador" de metales pesados fue enunciado la primera vez por Roger D. Reeves y surgió a partir del descubrimiento de altas concentraciones de $\mathrm{Ni}$ en la especie Sebertia acuminata Pierre ex Baill (ahora Niemeyera acuminata (Pierre ex Baill.) T. D. Penn) en Nueva Caledonia (Jaffré et al., 1976). El término "hiperacumulación" fue descrito un año más tarde por Robert R. Brooks como la acumulación de concentraciones de Ni superiores a $1000 \mu \mathrm{g} \mathrm{g}^{-1}$ en la materia seca de las hojas. También definió que las especies capaces de acumular más de 10000 $\mu \mathrm{g} \mathrm{g}^{-1}$ se denominaban hiperniqueloforas (Brooks et al., 1977). Las plantas clasificadas como hiperacumuladoras de $\mathrm{Ni}$ se caracterizaron por contener concentraciones de Ni entre 100 y 1000 veces mayores a las plantas que habitan en suelos no metalíferos, y entre 10 y 100 veces mayores a las presentes sobre suelos ricos en $\mathrm{Ni}$ formados sobre roca ultramáfica. Si bien hubo cierta arbitrariedad en la elección de este criterio, se observó en 
varias formaciones vegetales que las concentraciones de $\mathrm{Ni}$ entre 100 y $1000 \mathrm{\mu g} \mathrm{g}^{-1}$ son escasas, mientras que las superiores a $1000 \mathrm{\mu g} \mathrm{g}^{-1}$ parecen representar una respuesta diferente de las plantas basada en funciones metabólicas características e inusuales (van der Ent et al., 2013).

El concepto de hiperacumulación de $\mathrm{Ni}$ fue reformulado por Reeves (1992), quien planteó que una especie hiperacumuladora de $\mathrm{Ni}$ es aquella que acumula más de $1000 \mathrm{~g} \mathrm{~g}^{-1}$ del metal en la materia seca de su parte aérea, en al menos un espécimen que crece en su hábitat. La especificación sobre la localización del $\mathrm{Ni}$ en la parte aérea de la planta, obedece a la dificultad para eliminar el suelo de las muestras de raíces y a que la inmovilización de metales en las raíces es un proceso común (Baker, 1981). Cuando se estableció que el proceso de hiperacumulación ocurre solo en plantas que crecen en su hábitat, queda- ron fuera del concepto las que se cultivan en sustratos artificiales, como los suelos enriquecidos con metales o el cultivo en soluciones nutritivas. Además, las plantas deben crecer de forma saludable, ser capaces de reproducirse y mantener una población autosostenida (van der Ent et al., 2013).

A partir de la identificación de varias hiperacumuladoras de $\mathrm{Ni}$ en diferentes regiones del mundo, el término se aplicó a especies que acumulan otros metales, para los cuales se definieron diferentes concentraciones umbrales (Tabla 1). Muchas especies (721) hiperacumulan metales pesados, metaloides y tierras raras como: Ni (523), Cu (53), Co (42), Cr (1), $\mathrm{Mn}$ (42), Zn (20), Pb (8), Cd (7), Se (41), TI (2), As (5), La (1), y Ce (1). Algunas especies son capaces de hiperacumular más de un elemento químico (Reeves et al., 2017).

Tabla 1. Concentración umbral para las especies hiperacumuladoras de metales.

\begin{tabular}{cc}
\hline Metal & Concentración $\left(\boldsymbol{\mu g ~ g ^ { - 1 } )}\right.$ \\
\hline $\mathrm{Cu}$ & 300 \\
\hline $\mathrm{Co}$ & 300 \\
\hline $\mathrm{Zn}$ & 3000 \\
\hline $\mathrm{Mn}$ & 10000 \\
\hline $\mathrm{Cd}$ & 100 \\
\hline $\mathrm{Pb}$ & 1000 \\
\hline $\mathrm{Cr}$ & 300 \\
\hline
\end{tabular}

Para el Cu y el Co se definió inicialmente un valor superior a $1000 \mathrm{\mu g} \mathrm{g}^{-1}$, como para el $\mathrm{Ni}$, de acuerdo con valores obtenidos a partir de especies que crecen en la República Democrática del Congo. Estos valores no se pudieron confirmar en estudios más recientes y el error se atribuyó a la presencia de polvo en las muestras vegetales, por lo que se consideró que este valor era muy alto para definir la hiperacumulación de Cu (Faucon et al., 2007). La identificación de especies hiperacumuladoras de Co ha 
presentado una dificultad similar a la del $\mathrm{Cu}$ y por ello se estableció el valor de $300 \mu \mathrm{g} \mathrm{g}^{-1}$ para ambos metales (Krämer, 2010).

La hiperacumulación de Zn se estableció para plantas que superen el valor de $10000 \mathrm{\mu g} \mathrm{g}^{-1}$ (Baker y Brooks, 1989), pero esos mismos autores consideraron años más tarde que el valor era indebidamente restrictivo (Reeves y Baker, 2000). Para el Zn, un elemento que se encuentra normalmente a concentraciones entre $50-500 \mu^{-1} ~ g^{-1}$, una concentración de $3000 \mu \mathrm{g} \mathrm{g}^{-1}$ es más que notable y merecedora de ser tomada para establecer la hiperacumulación de este metal (Krämer, 2010).

El $\mathrm{Mn}$ se acumula en varias especies del género Maytenus, algunas que crecen en los suelos formados sobre rocas ultramáficas en Nueva Caledonia acumulan más de $10000 \mu \mathrm{g} \mathrm{g}^{-1}$ de Mn (Jaffré, 1977). En un inicio, estas especies fueron clasificadas como hipermanganesoforas lo que hizo suponer $1000 \mathrm{\mu g} \mathrm{g}^{-1}$ como criterio para la hiperacumulación del metal, pero los autores consideraron $10000 \mu \mathrm{g} \mathrm{g}^{-1}$ como la concentración umbral para este criterio (Baker y Brooks, 1989).

La hiperacumulación de Cd se informó por primera vez en la especie Thlaspi caerulescens J. Presl y C. Presl (ahora Noccaea caerulescens F. K. Mey). La tendencia de esta especie a hiperacumular Cd varía ampliamente entre las poblaciones (Lombi et al., 2001; Assuncao et al., 2003; Roosens et al., 2003). La inmensa mayoría de los suelos naturales ricos en $\mathrm{Cd}$ (suelos de tipo Calamina) son también ricos en $\mathrm{Pb}$ y Zn. Por lo tanto, muchas de la hiperacumuladoras de Cd también hiperacumulan Zn o al menos son tolerantes a este metal; lo mismo sucede con el $\mathrm{Pb}$. La especie Arabis paniculata Franch. de China acu- mula $20800 \mu \mathrm{g} \mathrm{g}^{-1}$ de Zn, así como 2300 $\mu \mathrm{g} \mathrm{g}^{-1}$ de $\mathrm{Pb}$ y $434 \mu \mathrm{g} \mathrm{g}^{-1}$ de $\mathrm{Cd}$ (Tang et al., 2009).

Varias especies acumulan concentraciones superiores a $1000 \mu \mathrm{g} \mathrm{g}^{-1}$ de $\mathrm{Pb}$ en la materia seca de sus hojas, lo mismo en su hábitat que en solución nutritiva, como el ecotipo de $N$. caerulescens que crece en poblaciones al sur de Francia, (Mohtadi et al., 2012). Concentraciones foliares muy altas se detectaron en Brassica juncea (L.) Czern. y Brassica carinata A. Braun, mediante la aplicación de sustancias movilizadoras y que forman complejos de $\mathrm{Pb}$, como el EDTA (ácido etilendiaminotetraacético) y el EDDS (ácido etilendiamino-N, N'-disuccínico), tanto en solución nutritiva como en suelo tratado con los agentes quelantes (Vassil et al., 1998). Esta técnica se denomina "Fitoextracción in situ inducida por quelantes", es excesivamente cara para la aplicación a gran escala y genera problemas medioambientales serios. Los agentes quelantes que se han utilizados movilizan el $\mathrm{Pb}$ y provoca su lixiviación, por lo que contaminan las aguas subterráneas y los suelos circundantes, lo que generó un escenario de contaminación casi imposible de controlar (Chaney et al., 2007).

No existen evidencias claras de la hiperacumulación de $\mathrm{Cr}$ en las plantas. Este metal generalmente no tiene una alta biodisponibilidad en el suelo, y por tanto existe una baja absorción potencial para las plantas. Varias especies se han informado como hiperacumuladoras de $\mathrm{Cr}$, Leersia hexandra Sw. (Zhang et al., 2007) y Spartina argentinensis Parodi (Redondo-Gómez et al., 2011). Los valores de Cr detectados en las muestras diferían mucho entre los sitios y las plantas, lo que indica una posible contaminación de la superficie o la 
incapacidad de eliminar la contaminación superficial durante el análisis. Sobre la base de las bajas concentraciones de $\mathrm{Cr}$ en las plantas, tanto en suelos no metalizados $\left(<1 \mu \mathrm{gg}^{-1}\right)$ comoen losultramáficos $\left(<50 \mu \mathrm{gg}^{-1}\right)$, se propuso establecer el criterio de $300 \mathrm{\mu g} \mathrm{g} \mathrm{g}^{-1}$ para el estado de hiperacumulación (van der Ent et al., 2013).

\section{La hiperacumulación de Ni por las plantas}

A pesar de que el término "hiperacumulador" se estableció muchos años después, la primera especie hiperacumuladora de $\mathrm{Ni}$ (Alyssum bertolonii Desv.) se identificó en Italia a mediados del siglo pasado (Minguzzi y Vergnano, 1948). A partir de ese año se han identificado en el mundo 532 especies, que pertenecen a 130 géneros de 52 familias botánicas. La presencia de hiperacumuladoras de $\mathrm{Ni}$ se ha informado por varios países como: Nueva Caledonia (65), Turquía (59), Brasil (30) y Malasia (24), entre otros (Reeves et al., 2017).Cuba es el país con el mayor número de hiperacumuladoras de Ni con 130 especies. Las primeras especies cubanas informadas en la literatura son Leucocroton flavicans Müll. Arg. y Buxus flaviramea (Britton) Mathou (Berazaín, 1981). Posteriormente se informaron 128 nuevas especies pertenecientes a las familias Euphorbiaceae (38), Phyllanthaceae (24), Asteraceae (21), Buxaceae (16), Rubiaceae (10), Myrtaceae (6), Tiliaceae (5), Clusiaceae (4), Ocnaceae (2), Acanthaceae (1) y Oleaceae (1). La mayoría de estas especies solamente crecen en suelos formados sobre roca ultramáfica ("serpentina" obligadas) o también desarrollarse en otros tipos de suelo ("serpentina" facultativas) sin hiperacumular el metal (Reeves et al., 1996, 1999).

La familia Euphorbiaceae en Cuba está representada por 251 especies distribuidas en 37 géneros y 137 de ellas son endémicas
(Greuter y Rankin Rodríguez, 2016). Esta familia agrupa la mayor cantidad de hiperacumuladoras en la isla y el género más representativo es Leucocroton, donde las 26 son hiperacumuladoras (Jestrow et al., 2010). Veinte especies superan el $1 \%$ de $\mathrm{Ni}$ en sus hojas, lo que permite identificarlas como hiperniqueloforas y L. havanensis hiperacumula aproximadamente $20000 \mathrm{\mu g} \mathrm{g}^{-1}$ de $\mathrm{Ni}$ en sus hojas (Reeves et al., 1996).

\section{Importancia fisiológica y ecológica del $\mathrm{Ni}$ en las plantas}

El $\mathrm{Ni}$ es un nutriente esencial para las plantas superiores y desde 1970 se identificó como cofactor de la enzima ureasa en las plantas. La urea es una sustancia que se genera a partir del metabolismo celular y resulta tóxica a altas concentraciones (Fishbein et al., 1976). Tres rutas metabólicas sintetizan urea en las plantas superiores. Esta sustancia es un producto de la actividad de la arginasa mitocondrial en el ciclo de la ornitina. Por otra parte, las ureidas constituyen la principal reserva de nitrógeno en la familia Fabaceae, el ácido alantoínico se transloca de la raíz a los brotes y allí se hidroliza a glioxalato y urea mediante la actividad de la alantoicasa. También se identificó la síntesis de urea en la ruta metabólica de la canavanina, un aminoácido no proteico presente en el género Canavalia y en Soya (Glycine max L.) (Dixon et al., 1975; Welch, 1981).

La deficiencia del Ni disminuye la capacidad de desarrollar semillas viables en cebada (Hordeum vulgare L.), debido al insuficiente crecimiento del embrión. En estas plantas, la raíz del embrión crece pobremente o incluso permanece sin desarrollo alguno. Además se han informado muchas anomalías en el desarrollo del endospermo debido a la disminución de la 
actividad de las deshidrogenasas (Brown et al., 1987). La disminución de la actividad de la ureasa provoca la acumulación de urea hasta niveles tóxicos, que a su vez afecta la formación del embrión y retarda la germinación de la semilla en A. thaliana (Zonia et al., 1995).

La función ecológica de la hiperacumulación de $\mathrm{Ni}$ en las plantas no se ha utilizado exhaustivamente, pero seha especulado que pudiera ser una respuesta de tolerancia al metal, resistencia a la sequía, una estrategia competitiva e incluso una protección ante el ataque de bacterias, hongos y herbívoros. A pesar de la escasa información sobre el tema se demostró la inhibición total del crecimiento de Pseudomonas syringae Van Hall pv. maculicola M4 en hojas de N. caerulescens $\left(1400 \mu \mathrm{g} \mathrm{g}^{-1} \mathrm{de} \mathrm{Ni}\right)$. Esta bacteria es un patógeno natural de la especie y creció en plantas cultivadas en ausencia del metal (Fones et al., 2010). También se observó un alto porcentaje de mortalidad en larvas de Spodoptera exigua Hübner alimentadas con dietas artificiales que contenían $\mathrm{Ni}$. El efecto del metal aumentó, de forma aditiva, cuando se combinó con Co y $\mathrm{Cu}$, y de forma sinérgica cuando se combinó con $\mathrm{Zn}$. El efecto del $\mathrm{Ni}$ combinado con compuestos orgánicos también aumentó de forma aditiva, cuando se combinó con Nicotina y polvo de semillas de Mostaza, y de forma sinérgica cuando se combinó con ácido tánico (Cheruiyot et al., 2015).

\section{Ligandos involucrados en la desintoxicación de metales pesados}

La planta absorbe los iones metálicos a través de la raíz y los traslada por el xilema a los brotes, donde ocurren la desintoxicación y el almacenamiento en com- partimentos celulares. Estos procesos ocurren mediante transporte, quelación y secuestración por ligandos orgánicos a nivel subcelular. Los potenciales ligandos se agrupan para su estudio en tres grupos principales: (1) donantes de oxígeno (carboxilatos: malato, citrato, malonato, succinato y oxalato); (2) donantes azufre (metalotioneinas y fitoquelatinas) y donantes de nitrógeno (aminoácidos) (Clemens, 2001). El acomplejamiento de los metales con estos ligandos disminuye la actividad de los iones libres y así se reduce la toxicidad; posteriormente se localizan en el apoplasto (Vázquez et al., 1992), se confinan en las vacuolas de las células epidérmicas o se unen a la pared celular (Krämer et al., 2000).

Los ligandos tienen una función muy importante en la disminución del efecto nocivo de los iones metálicos libres en la célula, la especificidad del ion por el ligando está muy influenciada por el pH. El Ni parece estar asociado a moléculas polares de baja masa molecular (aminoácidos y ácidos orgánicos) y no a moléculas de alto peso molecular como las fitoquelatinas $y$ metalotioneínas. A pH ácido, los ácidos orgánicos son mejores agentes quelantes de $\mathrm{Ni}$ que los aminoácidos, pero si el pH aumenta, también aumenta la competencia por el aminoácido y desplaza a los ácidos orgánicos (Homer et al., 1997).

Las fitoquelatinas son polipéptidos sintetizados por la fitoquelatina sintasa a partir del glutatión (Grill et al., 1987) y son además análogos funcionales de las metalotioneínas, proteínas de bajo peso molecular ricas en cisteína, que tienen la función en varias especies vegetales de neutralizar el efecto del $\mathrm{Cd}$ en las células (Wagner y Trotter, 1982). 


\section{Leucocroton havanensis}

L. havanensis es una especie hiperacumuladora de Ni endémica de Cuba. Esta planta habita en el matorral xeromorfo espinoso sobre "suelo serpentina" (cuabales) de las áreas protegidas "La Coca", provincia La Habana y "Lomas de Galindo" en la provincia Mayabeque (Reeves et al., 1999). El término "suelo serpentina", que se utiliza ampliamente para definir el sustrato formado sobre roca ultramáfica, no existe como tipo de suelo. Aunque se caracteriza por contener la roca serpentinita (láminas verde-azules), suelen ser ferríticos y ferralíticos rojos (Lewis et al., 2006). La naturaleza química extrema de estos suelos metalíferos, debido a las altas concentraciones de $\mathrm{Ni}, \mathrm{Cr}, \mathrm{Co}, \mathrm{Mn}, \mathrm{Mg}$ y Fe, y las bajas concentraciones de nutrientes esenciales para el crecimiento de las plantas como N, P, $\mathrm{K}$ y $\mathrm{Ca}$, hacen que estas áreas alberguen una flora característica con un alto número de especies endémicas (Borhidi, 1988). Las 26 especies identificadas en el género Leucocroton son hiperacumuladoras de $\mathrm{Ni}$, mientras que las especies cercanas Lasiocroton microphyllus y Lasiocroton leprosus Jestrow (antes Leucocroton microphyllus (A. Rich.) Pax y Hoffm. de Cuba y Leucocroton leprosus Willd Pax y Hoffm. de Haití) no hiperacumulan el metal.

L. havanensis es una especie clasificada como amenazada en la Lista Roja de la Flora de Cuba (González Torres et al., 2016), debido a la reducción del tamaño de sus poblaciones y a que su hábitat se ha modificado por la acción del hombre. En el área que hoy ocupan ambas reservas ecológicas, se construyó una presa y alrededor de ella habita una población rural que realiza actividades agrícolas y ganaderas. Varios taxones introducidos (invasoras) compiten por el espacio como el Marabú
(Dichrostachys cinerea (L.) Wight y Arn.) y el Weiler (Mimosa pellita Kunth ex Willd.), entre otros (Herrera Oliver et al., 1987).

\section{Clasificación taxonómica}

Leucocroton havanensis Borhidi; Eukaryota; Viridiplantae; Streptophyta; Embryophyta; Tracheophyta; Spermatophyta; Magnoliophyta; eudicotyledons; core eudicotyledons; rosids; eurosids I; Malpighiales; Euphorbiaceae; Acalyphoideae; Adelieae; Leucocroton (Webster, 1975; Byng et al., 2016).

\section{Descripción botánica}

Según la descripción original que aparece en la Flora de Cuba: Leucocroton havanensis Borhidi; Leucocroton flavicans Muell. Arg.; Leucocroton angustifolius Pax y Hoffm. - "Cuaba amarilla". - Arbustos de hasta $5 \mathrm{~m}$; pecíolo de 5-10 mm, canaliculado arriba; hojas lanceoladas a lineal-lanceoladas, agudas a mucronadas en el ápice, estrechas en la base, de 5 a $13 \mathrm{~cm}$ y 1-2,5 de ancho, brillantes y finamente reticuladas, el nervio medio hundido en el haz, el envés blancuzco, estrellado-tomentoso y el nervio medio prominente. Flores masculinas aglomeradas en espigas cortas, pedunculadas; sépalos cuatro, triangulares tomentosos; estambres exertos, $10 \pm$; ovario rudimentario peloso; inflorescencia hermafrodita con 1 flor en la extremidad; sépalos lanceolados; estilos cortos, muy divididos; cápsula de $5 \mathrm{~mm}$. - Cuabales: Matanzas, Habana - Endémica (León y Alain, 1953).

\section{CONCLUSIONES}

Las plantas hiperacumuladoras de metales pueden tener varias aplicaciones en la alimentación y la industria. Estas especies vegetales pueden utilizarse como suplemento nutricional para la producción de 
biofortificantes ricos en elementos como Zn y Se, en ocasiones deficitarios en la dieta y muy importantes en el metabolismo de los organismos vivos.

Debido a la capacidad que poseen estas plantas de crecer en suelos mineralizados, pueden utilizarse en proyectos de rehabilitación minera, para la recuperación de la capa vegetal en minas abandonadas.

Leucocroton havanensis es una especie que se puede emplear en la agrominería del $\mathrm{Ni}$, como una alternativa ecológica a la extracción convencional del metal, que erosiona el suelo y contamina el aire y los ríos.

\section{REFERENCIAS BIBLIOGRÁFICAS}

Assuncao, A. G. L., Bookum, W. M., Nelissen, H. J. M., Vooijs, R., Schat, H. y Ernst, W. H. O. (2003). Differential metal-specific tolerance and accumulation patterns among Thlaspi caerulescens populations originating from different soil types. New Phytol. 159, 411- 419. https://doi.org/10.1 046/j.1469-8137.2003.00819.

Baker, A. J. (1981). Accumulators and excluders-strategies in the response of plants to heavy metals. J. Plant Nutr. 3, 643-654.

Baker, A. J. M. y Brooks, R. R. (1989). Terrestrial higher plants which hyperaccumulate metallic elements: a review of their distribution, ecology and phytochemistry. Biorecovery 1: 81-126. Chilean case. Ambio $21,138-144$.

Berazaín, R., (1981). Sobre el endemismo de la florula serpentinicola de" Lomas de Galindo", Canasi, Habana. Rev. Jardín Botánico Nac. 29-47.
Borhidi, A., (1988). Efecto ecológico de la roca serpentina a la flora y vegetación de Cuba. Acta Bot Hung 34, 123-174.

Boyd, R. S. y Jhee, E. M. (2005). A test of elemental defence against slugs by $\mathrm{Ni}$ in hyperaccumulator and non-hyperaccumulator Streptanthus species. Chemoecology 15, 179-185.

Boyd, R. S., Shaw, J. J. y Martens, S. N. (1994). Nickel Hyperaccumulation Defends Streptanthus polygaloides (Brassicaceae) Against Pathogens. Am. J. Bot. 81, 294-300. https://doi. org/10.2307/2445455

Brooks, R. R., Lee, J., Reeves, R. D. y Jaffre, T. (1977). Detection of nickeliferous rocks by analysis of herbarium specimens of indicator plants. J. Geochem. Explor. 7, 49-57. https://doi.org/10.1016/03756742(77)90074-7

Brown, P. H., Welch, R. M. y Cary, E. E. (1987). Nickel: A micronutrient essential for higher plants. Plant Physiol. 85, 801-803.

Byng, J. W., Chase, M. W., Christenhusz, M. J., Fay, M. F., Judd, W. S., Mabberley, D. J., Sennikov, A. N., Soltis, D. E., Soltis, P. S. y Stevens, P. F. (2016). An update of the Angiosperm Phylogeny Group classification for the orders and families of flowering plants: APG IV. Bot. J. Linn. Soc. 181, 1-20.

Chaney, R. L., Angle, J. S., Broadhurst, C. L., Peters, C. A., Tappero, R. V. y Sparks, D. L. (2007). Improved Understanding of Hyperaccumulation Yields Commercial Phytoextraction and Phytomining Technologies. J. Environ. Qual. 36, 1429. https:// doi.org/10.2134/jeq2006.0514 
Chen, Y., Zhang, Y., Cheng, Q., Niu, M., Liang, H., Yan, H., Zhang, X., da Silva, J. A. T. y Ma, G. (2016). Plant regeneration via direct and callus-mediated organogenesis from leaf explants of Chirita swinglei (Merr.) WT Wang. Vitro Cell. Dev. Biol.-Plant $52,521-529$.

Cheruiyot, D. J., Boyd, R. S. y Moar, W. (2015). Testing the joint effects hypothesis of elemental defense using Spodoptera exigua. J. Chem. Ecol. 41, 168-177.

Clemens, S. (2001). Molecular mechanisms of plant metal tolerance and homeostasis. Planta 212, 475-486.

De Souza, R. A., Dantas, P. V. P., de Freitas Cavalcante, P., Tenório, R. R. y Houllou, L. M. (2017). Basic procedure for the in vitro propagation of Brazilian trees for reforestation purposes. J. Environ. Anal. Prog. 2, 107-114.

Dixon, N. E., Gazzola, C., Blakeley, R. L. y Zerner, B. (1975). Jack bean urease (EC 3.5. 1.5). Metalloenzyme. Simple biological role for nickel. J. Am. Chem. Soc. 97, 4131-4133.

Etienne, H., Bertrand, B., Dechamp, E., Maurel, P., Georget, F., Guyot, R. y Breitler, J. C. (2016). Are genetics and epigenetic instabilities of plant embryogenic cells a fatality? The experience of coffee somatic embryogenesis. Hum. Genet. Embryol. 6.

Faucon, M. P., Shutcha, M. N. y Meerts, P. (2007). Revisiting copper and cobalt concentrations in supposed hyperaccumulators from SC Africa: influence of washing and metal concentrations in soil. Plant Soil 301, 29-36.
Fishbein, W. N., Smith, M. J., Nagarajan, K.y Scurzi, W. (1976). 1ST Natural nickel metalloenzyme-urease, in: Federation Proceedings. FEDERATION AMER SOC EXP BIOL 9650 ROCKVILLE PIKE, BETHESDA, MD 20814-3998 USA, pp. 1680-1680.

Fones, H., Davis, C. A., Rico, A., Fang, F., Smith, J. A. C. y Preston, G. M. (2010). Metal hyperaccumulation armors plants against disease. PLoS Pathog. 6, e1001093.

Gautheret, R. J. (1934). Culture du tissu cambial. CR Hebd Seances Acad Sc 198, 2195-2196.

González, L. R., Palmarola, A., González, L., Bécquer, E. R., Testé, E., Barrios, D., Acosta, Z., Alomá, O., Álvarez, J. C. y Berazaín, R. C. (2016). Lista Roja de la Flora de Cuba 2016.

Greuter, W. y Rankin Rodríguez, R. (2016). Espermatófitos de Cuba. Inventario preliminar.

Grill, E., Winnacker, E. L. y Zenk, M. H. (1987). Phytochelatins, a class of heavy-metal-binding peptides from plants, are functionally analogous to metallothioneins. Proc. Natl. Acad. Sci. 84, 439-443.

Grover, A. y Sharma, P. C. (2014). Development and use of molecular markers: past and present. Crit. Rev. Biotechnol. 36, 290302.

Haberlandt, G., (1902). Cellular totipotency. Elsevier Science Publishing Co., New York.

Herrera, P., Montes, L. y Chiappy, C. (1987). Valoración Botánica de la propuesta de reserva natural" Loma de la Coca", Ciudad de La Habana, Cuba. Acta Bot Cuba. 
Homer, F. A., Reeves, R. D. y Brooks, R. R. (1997). The possible involvement of aminoacids in nickel chelation in some nickel-accumulating plants. Curr Top Phytochem 14, 31-33.

Izquierdo-Oviedo, H., Disotuar, R., González, M. C. y González, S. J. (2016). Micropropagation of garlic (Allium sativum L.) and determination of the genetic stability of the plantlets obtained by AFLP markers. Biotecnol. Apl. 33, 4211-4218.

Jaffré, T. (1977). Accumulation du manganèse par des especes associées aux terrains ultrabasiques de Nouvelle-Calédonie. Cr Acad Sci Paris D 284, 1573-5.

Jaffré, T., Brooks, R. R., Lee, J. y Reeves, R. D. (1976). Sebertia acuminata: a hyperaccumulator of nickel from New Caledonia. Science 193, 579-580.

Jestrow, B., Rodríguez, F. J. y Francisco-Ortega, J. (2010). Generic delimitation in the Antillean adelieae (Euphorbiaceae) with description of the Hispaniolan endemic genus Garciadelia. Taxon 1801-1814.

Krämer, U. (2010). Metal Hyperaccumulation in Plants. Annu. Rev. Plant Biol. 61, 517-534. https://doi.org/10.1146/annurev-arplant-042809-112156

Krämer, U., Pickering, I. J., Prince, R. C., Raskin, I. y Salt, D. E. (2000). Subcellular localization and speciation of nickel in hyperaccumulator and non-accumulator Thlaspispecies. Plant Physiol. 122, 1343-1354.

Kumar, R. K., Chu, H. H., Abundis, C., Vasques, K., Rodriguez, D. C., Chia, J.-C., Huang, R., Vatamaniuk, O. K. y Walker, E. L. (2017). Iron-Nicotianamine Transporters
Are Required for Proper Long Distance Iron Signaling. Plant Physiol. 175, 1254-1268.

Larkin, P. J. y Scowcroft, W. R. (1981). Somaclonal variation - a novel source of variability from cell cultures for plant improvement. Theor. Appl. Genet. 60, 197-214. https://doi.org/10.1007/BF0234 2540

Lee, J. y Pijut, P. (2017). Adventitious shoot regeneration from in vitro leaf explants of Fraxinus nigra. Plant Cell Tissue Organ Cult. PCTOC. https://doi.org/10.1007/s11240017-1228-1

León, H. y Alain, H. (1953). Flora de Cuba. Dicotiledóneas: Malpighiaceae a Myrtaceae. C. Habana 3.

Lewis, J. F., Draper, G., Espaillat, J., Proenza, J. A. y Jiménez, J. (2006). Ophiolite-related ultramafic rocks (serpentinites) in the Caribbean region: a review of their occurrence, composition, origin, emplacement and Ni-laterite soil formation. Geol. Acta Int. Earth Sci. J. 4, 237-264.

Lombi, E., Zhao, F. J., Mc Grath, S. P., Young, S. D. y Sacchi, G. A. (2001). Physiological evidence for a high-affinity cadmium transporter highly expressed in a Thlaspi caerulescens ecotype. New Phytol. 149, 53-60.

Mandal, A. B., Maiti, A., Chowdhury, B. y Elanchezhian, R. (2001). Isoenzyme markers in varietal identification of banana. Vitro Cell. Dev. Biol.-Plant 37, 599-604.

Minguzzi, C. y Vergnano, O. (1948). II contenuto di nichel nelle ceneri di Alyssum bertolonii Desv. Mem Soc Tosc Sci Nat Ser A $55,49-77$. 
Mohtadi, A., Ghaderian, S. M. y Schat, H. (2012). A comparison of lead accumulation and tolerance among heavy metal hyperaccumulating and non-hyperaccumulating metallophytes. Plant Soil 352, 267-276.

Redondo, S., Mateos, E., Vecino, I. y Feldman, S.R. (2011). Accumulation and tolerance characteristics of chromium in a cordgrass Cr-hyperaccumulator, Spartina argentinensis. J. Hazard. Mater. 185, 862-869.

Reeves, R. D. y Baker, A. J. (2000). Metal accumulating plants. Phytoremediation Toxic Met. Using Plants Clean Environ. Raskin Ensley BD Eds John Wiley Sons Inc N. Y.

Reeves, R. D., Baker, A. J., Jaffré, T., Erskine, P. D., Echevarria, G. y Ent, A. (2017). A global database for plants that hyperaccumulate metal and metalloid trace elements. New Phytol.

Reeves, R. D., Baker, A. J. M., Bgrhidi, A. y Berazaín, R. (1996). Nickel-accumulating plants from the ancient serpentine soils of Cuba. New Phytol. 133, 217-224. https://doi.org/10.1111/j.1469-8137.1996.tb01888.x

Reeves, R. D., Baker, A. J. M., Borhidi, A. y Berazain, R. (1999). Nickel hyperaccumulation in the serpentine flora of Cuba. Ann. Bot. 83, 29-38.

Roosens, N., Verbruggen, N., Meerts, P., Ximénez-Embún, P. y Smith, J. A. C. (2003). Natural variation in cadmium tolerance and its relationship to metal hyperaccumulation for seven populations of Thlaspi caerulescens from western Europe. Plant Cell Environ. 26, 1657-1672.

Tang, Y.-T., Qiu, R.-L., Zeng, X.-W., Ying, R.R., Yu, F.-M. y Zhou, X.-Y. (2009). Lead, zinc, cadmium hyperaccumulation and growth stimulation in Arabis paniculata Franch. Environ. Exp. Bot. 66, 126-134.

Van Der Ent, A., Baker, A. J., Reeves, R. D., PoIlard, A. J. y Schat, H. (2013). Hyperaccumulators of metal and metalloid trace elements: facts and fiction. Plant Soil 362, 319-334.

Vassil, A. D., Kapulnik, Y., Raskin, I. y Salt, D. E. (1998). The role of EDTA in lead transport and accumulation by Indian mustard. Plant Physiol. 117, 447-453.

Vázquez, M. D., Barceló, J., Poschenrieder, C. H., Madico, J., Hatton, P., Baker, A. J. M., Cope, G. H. y (1992). Localization of zinc and cadmium in Thlaspi caerulescens (Brassicaceae), a metallophyte that can hyperaccumulate both metals. J. Plant Physiol. 140, 350-355.

Ventura de Jesús J. (2018). Resaltan la importancia de la minería y la metalurgia para la economía cubana. Granma 2.

Wagner, G. J. y Trotter, M. M. (1982). Inducible cadmium binding complexes of cabbage and tobacco. Plant Physiol. 69, 804-809.

Webster, G. L. (1975). Conspectus of a new classification of the Euphorbiaceae. Taxon 593-601.

Welch, R. M. (1981). The biological significance of nickel. J. Plant Nutr. 3, 345-356.

Went, F. W. (1926). On growth-accelerating substances in the coleoptile of Avena sativa, in: Proc. Kon. Ned. Akad. Wet. p. 1.

Zhang, X.-H., Liu, J., Huang, H.-T., Chen, J., Zhu, Y.-N. y Wang, D.-Q. (2007). Chromium 
accumulation by the hyperaccumulator plant Leersia hexandra Swartz. Chemosphere 67, 1138-1143.

Zonia, L. E., Stebbins, N. E. y Polacco, J. C. (1995). Essential role of urease in germination of nitrogen-limited Arabidopsis thaliana seeds. Plant Physiol. 107, 1097-1103. 